\title{
Atypical Presentation of Sarcoidosis on ${ }^{18}$ F-FDG PET/CT
}

\author{
Prashant Jolepalem ${ }^{1}$, Katie Zanyk McLean ${ }^{2}$, and Ching-Yee Oliver Wong ${ }^{1}$ \\ ${ }^{I}$ Department of Diagnostic Radiology and Molecular Imaging, Oakland University William Beaumont School of Medicine and \\ Beaumont Health System, Royal Oak, Michigan; and ${ }^{2}$ Oakland University William Beaumont School of Medicine, \\ Rochester Hills, Michigan
}

On ${ }^{18} \mathrm{~F}$-FDG PET, inflammatory processes can be one of the most confounding factors for interpretation, particularly when the presentation of the process is atypical, as it was in the case of sarcoidosis reported here. Clinicians should be aware that sarcoidosis may mimic lung malignancies and lymphomas, regardless of typical or atypical appearance on ${ }^{18} \mathrm{~F}-\mathrm{FDG}$ PET.

Key Words: oncology; lymphoma; PET/CT; FDG; atypical; sarcoidosis

J Nucl Med Technol 2014; 42:114-115

DOI: $10.2967 /$ jnmt.113.132654

$\mathbf{T}$ he presence of inflammatory processes on ${ }^{18} \mathrm{~F}-\mathrm{FDG}$ PET can be one of the most confounding factors for interpretation. Sarcoidosis and other granulomatous processes in the lungs are one of the more common examples of this problem. Quantitative criteria as well as familiarity with the typical appearance of granulomatous disease has somewhat obviated this problem. This case demonstrates an example of how an atypical presentation of sarcoidosis can easily be mistaken for malignancy in a patient being evaluated with PET scanning for diagnostic purposes.

\section{CASE REPORT}

A 35-y-old woman with a history of multiple pulmonary emboli presented with dyspnea and chest pain. A subsequent CT study using a pulmonary embolism protocol did not demonstrate a pulmonary embolism but did detect several prominent mediastinal lymph nodes (Fig. 1). The size and appearance of the lymph nodes raised suspicion for lymphoma.

An ${ }^{18}$ F-FDG PET/CT scan was ordered for diagnostic and staging purposes (Fig. 2). The study was performed after injection with $555 \mathrm{MBq}$ of the radiotracer and a 90-min uptake time. The whole-body PET images were obtained for $3 \mathrm{~min}$ per bed position, with 6 positions to cover from the base of the skull to the middle of the thighs,

\footnotetext{
Received Sep. 16, 2013; revision accepted Oct. 22, 2013.

For correspondence or reprints contact: Prashant Jolepalem, William

Beaumont Hospital, 3601 W. 13 Mile Rd., Royal Oak, MI 48073.

E-mail: pjolepalem@gmail.com

Published online Mar. 13, 2014.

COPYRIGHT (c) 2014 by the Society of Nuclear Medicine and Molecular Imaging, Inc.
}

using a 3-slice overlap. The images were acquired using the 3-dimensional high-sensitivity mode with an axial field of view in a $256 \times 256$ matrix. Then, iterative image reconstruction was performed on a $128 \times 128$ matrix using an ordered-subset expectation maximization algorithm for 30 subsets and 2 iterations, with a 7.0-mm postreconstruction filter. Concomitant CT data were used for attenuation correction using $140 \mathrm{kVp}, 120-200 \mathrm{~mA}$, and a 3-mm slice thickness.

The images demonstrated abnormal multifocal ${ }^{18} \mathrm{~F}-\mathrm{FDG}$ uptake only in the right suprahilar (maximum standardized uptake value $\left.\left[\mathrm{SUV}_{\max }\right], 16\right)$, right paratracheal $\left(\mathrm{SUV}_{\max }\right.$, 23), and subcarinal (SUV $\left.{ }_{\max }, 21\right)$ lymph nodes, with likely station 11 and 12 lymph nodes $\left(\mathrm{SUV}_{\max }, 11\right)$. This uptake followed a lymphatic drainage pattern suggestive of a pulmonary neoplasm in the right upper lobe. However, there was no evidence of an ${ }^{18} \mathrm{~F}-\mathrm{FDG}$-avid parenchymal lesion, leaving open the possibility of lymphoma despite the lack of lymph conglomeration that is typical of such high-grade ${ }^{18}$ F-FDG activity. Nonetheless, tissue sampling was indicated, and subsequent biopsy of the subcarinal lymph node was consistent with sarcoidosis.

\section{DISCUSSION}

${ }^{18}$ F-FDG uptake can be nonspecific and is associated with a variety of conditions, including infectious processes (pneumonia), chronic inflammatory processes (granulomatous disease), or acute inflammatory processes (postradiation changes) (1). These inflammatory conditions have in common the increased ${ }^{18} \mathrm{~F}-\mathrm{FDG}$ uptake by neutrophils, macrophages, and lymphocytes that may mimic neoplastic ${ }^{18}$ F-FDG uptake (2). These conditions may be indistinguishable from malignancy on the basis of PET alone. The ${ }^{18} \mathrm{~F}$-FDG PET study in this case demonstrated several discrete high-grade ${ }^{18} \mathrm{~F}$-FDG-avid lymph nodes in the subcarinal, right paratracheal, and right suprahilar lymph nodes, with likely station 11 and 12 lymph nodes.

The appearance of several discrete, intensely avid lymph nodes is not typical of lymphoma, because cases with such high-grade activity $\left(\mathrm{SUV}_{\max }>10\right)$ typically present with lymph node conglomerations. Additionally, other clusters of lymph nodes are typically ${ }^{18} \mathrm{~F}-\mathrm{FDG}-\mathrm{avid}$, such as the axillary and cervical nodes (3).

The anatomic distribution of the ${ }^{18}$ F-FDG-avid lymph nodes was consistent with the drainage pattern of a right- 


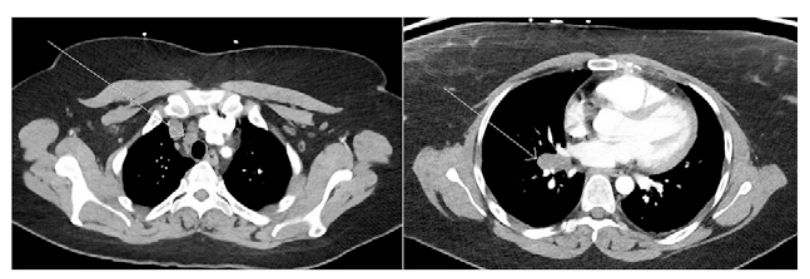

FIGURE 1. Contrast-enhanced CT scan demonstrating lymphadenopathy in right high paratracheal (left side) and right hilar (right side) lymph nodes (arrows).

upper-lobe neoplasm, as the malignant cells typically deposit into the right paratracheal lymph nodes and may skip stations 10-14 (4). Nevertheless, an ${ }^{18}$ F-FDG-avid neoplasm was not identified on the imaging, suggesting another etiology.

Activated granulomatous disease (histoplasmosis or sarcoidosis) or pneumonia is often a consideration in cases with a similar appearance. Although pneumonia will also follow anatomic lymph node drainage, the activity is often higher peripherally and decreases in the central lymph drainage sites, as is the case with lung cancer $(1,4)$. Additionally, there was no anatomic evidence of a consolidative or infiltrative process. Conversely, sarcoidosis will typically demonstrate higher activity in the central mediastinal nodes than in the hilar and lobar nodes, will affect both sides of the chest symmetrically, will not follow any specific lymph node drainage, and often does not present with a parenchymal lung lesion. Chundru et al. (1) proposed criteria based on case reviews to distinguish sarcoid from malignancy, including symmetric uptake in the hila; calcifications in the lymph nodes, liver, or spleen; and unexpected lymphatic drainage. However, the presentation of the lesions in this case did not meet any of these criteria. Therefore, the histopathologic diagnosis of sarcoidosis was unexpected.

${ }^{18} \mathrm{~F}-\mathrm{FDG}$ PET/CT has many proven applications beyond cancer imaging; its superior anatomic localization of increased metabolic activity has been found to be more sensitive for inflammatory or infectious processes such as sarcoidosis than are other nuclear imaging techniques such as ${ }^{67} \mathrm{Ga}$ imaging (2). However, the presence of such metabolically active benign processes can present a challenge when one interprets PET in the setting of cancer. Despite many studies using various criteria and semiquantitative methods to predict tumor phenotype or distinguish benign from malignant findings, atypical presentations (such as sarcoid in this case) are always possibilities that should be considered. Even as the accuracy of ${ }^{18}$ F-FDG PET imaging improves, its findings should always be considered in

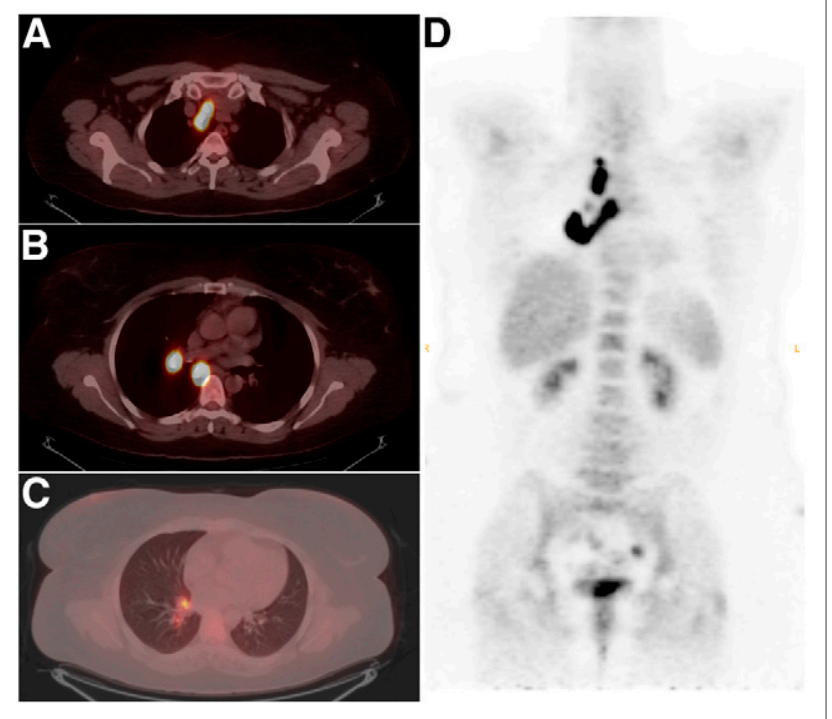

FIGURE 2. (A-C) Cross sections of PET/CT images showing intensely ${ }^{18} \mathrm{~F}-\mathrm{FDG}$-avid lymph nodes in right high paratracheal (A), right hilar and subcarinal (B), and interlobar and lobar (C) regions. (D) Coronal PET image demonstrating distribution pattern of metabolically active lymph nodes.

conjunction with the clinical setting and confirmed with tissue sampling when possible.

\section{CONCLUSION}

Interpretation of ${ }^{18} \mathrm{~F}$-FDG PET scans can be complex because of the multiple etiologies of increased ${ }^{18} \mathrm{~F}$-FDG uptake, including neoplasms, infectious conditions, and noninfectious inflammatory conditions. Clinicians should be aware that sarcoidosis may mimic lung malignancies and lymphomas, regardless of typical or atypical appearance on ${ }^{18} \mathrm{~F}-\mathrm{FDG}$ PET.

\section{DISCLOSURE}

No potential conflict of interest relevant to this article was reported.

\section{REFERENCES}

1. Chundru S, Wong CY, Wu D, et al. Granulomatous disease: is it a nuisance or an asset during PET/computed tomography evaluation of lung cancers? Nucl Med Commun 2008;29:623-627.

2. Braun JJ, Kessler R, Constantinesco A, Imperiale A. ${ }^{18}$ F-FDG PET/CT in sarcoidosis management: review and report of 20 cases. Eur J Nucl Med Mol Imaging 2008;35:1537-1543.

3. Ngeow JYY, Quek RHH, Ng DCE, et al. High SUV uptake on FDG-PET/CT predicts for an aggressive B-cell lymphoma in a prospective study of primary FDG-PET/CT staging in lymphoma. Ann Oncol 2009;20:1543-1547.

4. UyBico SJ, Wu CC, Suh RD, Le NH, Brown K, Krishnam MS. Lung cancer staging essentials: the new TNM staging system and potential imaging pitfalls. Radiographics 2010;30:1163-1181. 